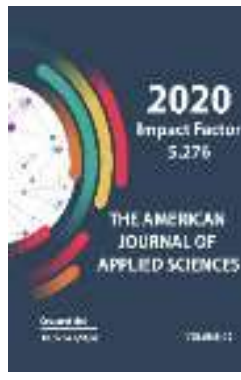

Journal Website: http://usajournalshub.c om/index,php/tajas

Copyright: Original content from this work may be used under the terms of the creative commons attributes 4.0 licence.

\section{Transparent Surface Lens Of Low-Temperature Solar Devices}

\author{
Jo'raev M.Q \\ Bukhara Engineering Technological Institute, Bukhara, Uzbekistan \\ Muzaffarov F.F \\ Bukhara Engineering Technological Institute, Bukhara, Uzbekistan \\ Rustamov S.Sh \\ Bukhara Engineering Technological Institute, Bukhara, Uzbekistan \\ Timirov H.N \\ Bukhara Engineering Technological Institute, Bukhara, Uzbekistan \\ Yakubov P,E \\ Bukhara Engineering Technological Institute, Bukhara, Uzbekistan
}

\title{
ABSTRACT
}

The transparent surface of low-temperature solar devices is insulated with glass or polyethylene. Ensuring more light penetration from such surfaces ensures efficient operation of the device, in other words, the device has a high efficiency. Selecting the device design to allow most of the sunlight falling on the transparent surface to enter the device, keeping the transparent surface clean, reducing the angle of incidence of the light to small values, and so on. In most cases, it depends on the parameters. In this article, we analyze the theoretical and practical solutions of work related to the selection and optimization of the angle of incidence of light on the transparent surface of the device to increase the incidence of sunlight during the day. Light absorption, rotation and absorotion of light into lowtemperature solar cells. Mathematical analysis and formulas are given.

\section{KEYWORDS}

Light wave length, effective return coefficient, effective ingestion coefficient, Buger-Lamber's law, the weaking of light. 


\section{INTRODUCTION}

As sunlight passes through the glass surface of the device, part of it passes, another part returns from the glass, and another part is absorbed by the glass material (Figure 1).

$$
E_{0}=E_{o^{\prime} t}+E_{q a y t}+E_{y u t}(1)
$$

where: $E_{0}$ - the intensity of light incident on the glass surface, $E_{o^{\prime} t}{ }^{-}$the intensity of light passing through the glass surface, $E_{\text {qayt }}$ - the intensity of light returning from the glass surface, $E_{y u t}$ - the intensity of light absorbed through the glass.The absorption of light in glass by the passage of sunlight through glass insulation of thickness $\delta$ can be determined according to Buger's-Lamber law.

$$
E_{y u t}=E_{0} \cdot e^{-k \delta}(2)
$$

where; $k$ is the attenuation coefficient of the beam:The value of this coefficient depends on the chemical nature of the transparent object and the wavelength $(\lambda)$ of the light incident on it.

\section{MATERIALS AND METHODS}

Experiments are usually performed to find the attenuation coefficient (k). In this article, we determine the value of this coefficient for ordinary window glass, which is widely used in solar devices.It is known that when light passes through a transparent object, the coefficients of the light passing through the glass, returning from the glass and absorbed by the glass in relation to the incident light are as follows:

The coefficient of reflected light: $\rho^{q a y t}=\frac{E_{\text {qayt }}}{E_{0}}$

The coefficient of absorbed light:

$$
\alpha^{y u t}=\frac{E_{y u t}}{E_{0}}
$$

Coefficient of transmitted light: $\tau^{o^{\prime} t}=\frac{E_{o^{\prime} t}}{E_{0}}$ 


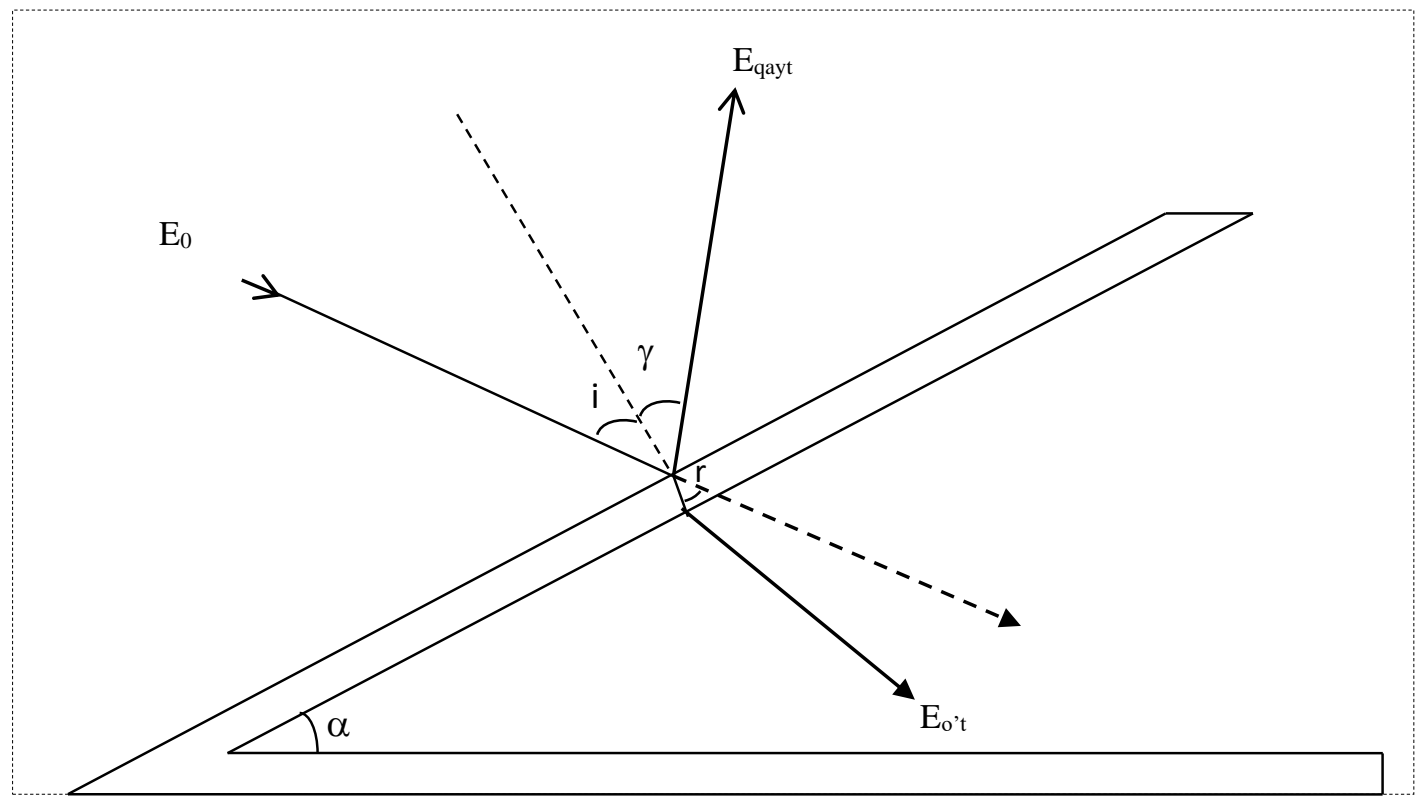

(Figure 1)

Here the angle of incidence of the i-beam on the glass surface; the angle of refraction of $r$ light as it passes through the glass surface; $\alpha$ is the angle formed by the glass with the horizon; The light can pass through glass of thickness $\delta$ and return to the surface several times. As can be seen from Figure 1, the values of the return rays and the transition rays must be taken into account. Therefore, the effective return coefficient $\left(\rho_{\text {eff }}\right)$, the effective absorption coefficient $\left(\alpha_{e f f}\right)$ and the effective transition coefficients $\left(\tau_{\text {eff }}\right)$ must be taken into account. The sum of all of them is 1 .

$$
\rho_{\text {eff }}+\alpha_{\text {eff }}+\tau_{\text {eff }}=1 .
$$

How much of the energy in each part makes up the total amount of energy $\left(E_{0}\right)$ can be determined using formula 3.The effective return factor can be determined as follows:

$$
\rho_{\text {eff }}^{q a y t}=\left[1+\frac{(1-\rho)^{2}(1-\alpha)^{2}}{(1+\rho)^{2}(1+\alpha)^{2}}\right],(4)
$$

here

$\rho-$ the coefficient of reflection of light from the glass. $\alpha$ the absorption coefficient of a-bottle.

The reflection coefficient can also be expressed by the angles of incidence and refraction of the beam.

$$
\rho=0.5\left[\frac{\sin (r-i)^{2}}{\sin (r+i)^{2}}+\frac{\tan (r-i)^{2}}{\tan (r+i)^{2}}\right],
$$

Here:

$r$ is the angle of refraction of light passing through the glass surface. It depends on the refractive index $n$ of the medium and the angle of incidence.

$$
r=\arcsin \left(\frac{\sin (i)}{n}\right),
$$

The absorption coefficient of light passing through the glass depends on the thickness of the glass.

(2) from the formula $\alpha=1-\frac{\cos (r)}{k \delta}(1-$

$$
\left.e^{\frac{-k \delta}{\cos (r)}}\right)
$$

This expression was suggested by Professor R. Avezov. Equation $\alpha=1-e^{\frac{-k \delta}{\cos (r)}}$ can be used instead. However, the degree of accuracy of 
expression (7) is much higher. It should be noted that formula (7) was determined by Professor R. Avezov by solving the thermal and optical equations of a transparent lighttransmitting surface. Effective absorption coefficient of transparent surface:

$$
\alpha_{\text {eff }}^{\text {yut }}=\alpha \frac{(1-\rho)}{1-\rho(1-\alpha)},
$$

Efficient conversion factor:

$$
\tau_{e f f}=\frac{(1-\rho)^{2}(1-\alpha)}{(1+\rho)(1-\alpha)^{2}},
$$

To calculate the above quantities, you need to know the angle of incidence of the light on the glass surface. To solve the problem, let's start with the vertical, i.e. $i=0$ and $r=0$. We find the extraction coefficient (attenuation of light).

$$
k=\frac{1}{\delta} \ln \frac{\tau_{e f f}}{(1-\rho)^{2}},
$$

\section{RESULT AND DISCUSSION}

Experimentally determined $\tau_{\text {eff }}=0,815$ and $\mathrm{i}=$ $0, n=1.526$ for glass, for glass thickness we find the following.

$$
\begin{gathered}
\rho=\left(\frac{1-n}{1+n}\right)^{2}=0.043 \\
k=\frac{1}{0.004} \ln \frac{0.815}{0.9151}=\frac{1}{29} m
\end{gathered}
$$

However, the value of $\tau_{\text {eff }}$ varies at different angles of incidence of light on the glass surface. The dependence of this value on the angle of incidence can be determined experimentally. If we determine the value of the return coefficient $\rho$ using formula (4), we get $\rho_{\text {eff }}$ values $\rho_{\text {eff }}=0,1$ and from formula (3) $\alpha_{\text {eff }}=0.085$. So the value of the quantities we are looking for is:

Effective return coefficient- $\rho_{\text {eff }}=0,1$
Effective transition coefficient- $\tau_{\text {eff }}=0,815$

Effective absorption coefficient- ${ }^{\mathrm{i}-} \alpha_{\text {eff }}=$ 0.085

\section{CONCLUSION}

Therefore, for an ordinary window glass with $\delta=4 \mathrm{~mm}$ refractive index $\mathrm{n}=1,526$, the extraction coefficient can be taken as $k=1$ / 29m. However, for cases where the thickness and refractive index are different and the angle of incidence of the beam is different, the calculation of the effective transition coefficient can be determined on the basis of this method. will be. In this case, the active life of each solar device is obtained. For example, to ensure that solar greenhouses receive enough sunlight in December, January, and February, the average, monthly arithmetic value of the coefficients of effective value

$\tau_{\text {eff }}$ for each day of these months and through them for each month must be determined. . It does not require complex work from the designer. At each time interval, it is sufficient to measure the amount of sunlight entering the device and the intensity of the sun falling on the transparent surface. The measuring instrument does not have to be very accurate and well calibrated. Because the ratio of these values is obtained.

$$
\tau_{\text {eff }}=\frac{E_{o^{\prime} t}}{E_{\mathrm{O}}}
$$

The extraction coefficient $\mathrm{k}$ depends on the angle of incidence and the angle of refraction of the transparent material thickness $\delta$.

\section{REFERENCES}

1. B.M. Yarovskiy, A.A. Detlaf Guide to Physics.

«Nauka» Fizike-Matematicheskaya

literatura.M 1985 y 
2. E.. A. Krasnoshchekov, A.S. Sukomel. "Zadachnik po teplopredache" Moscow "Energiya". 1980.

3. Use of solar energy. 1-collection under the editorship of d.t.nA.I Baumana.Moscow "Energy". 1957. 